\title{
Nash, bargaining and evolution
}

\begin{abstract}
Evolutionary accounts of morality tend to consider behavior in rather simple scenarios. For instance, evolutionary work on fairness focuses on the division of a windfall and, importantly, assumes the position of those involved are entirely symmetric. We consider more complicated strategic settings and find that there is a strong tendency for evolution to produce divisions consistent with the so-called Nash bargaining solution. We also uncover the evolutionary importance of comprehensiveness, an often-overlooked feature of division problems.
\end{abstract}

\section{Introduction}

One aim of the scientific approach to ethics is to provide a compelling evolutionary - be it biological or cultural - explanation of our sense of fairness. This is an extraordinarily difficult task because knowledge of the evolutionary past and the factors that shaped human sociality are, at best, opaque. Furthermore, the explanandum (our moral intuition) is difficult to pin down. Individuals may not possess clear and stable intuitions regarding ethical and social matters, and much experimental work indicates that philosophical intuitions can be easily manipulated.

Despite these two worries, prior work in evolutionary ethics has made significant headway. In addressing the first concern - our incomplete understanding of the past - theorists often deliberately abstract away from historical details and instead aim for explanations that focus only on 'payoffs the agents will obtain, contingent upon their action' (Ernst, 2005). This approach is compelling because it allows for a highly general account of behavior that can be mapped to various disparate phenomena. With respect to the second concern - intuitional (in)stability theorists have primarily limited their attention to those simple cases where our intuitions are resoundingly clear. Brian Skyrms, for instance, focuses on the case where two individuals must determine how to divide a windfall of money. The position of the two individuals are entirely symmetric: no one is more deserving or needy. An even split strikes us as 'fair', and psychological surveys indicate that this intuition is widely held (Nydegger \& Owen, 1974). Furthermore, the equal split is evolutionarily significant. Relying on a plethora of simple evolutionary models, Skyrms and his co-authors have shown that when symmetrically-situated individuals must divide a windfall, an adaptive process will push the population to the equal split. Evolution leads to outcomes we recognize as fair.

The goal of this paper is to explore a similarly spartan evolutionary explanation of our moral sense. Like prior investigations, we draw on game-theoretic methods but, importantly, look beyond those cases involving symmetrically-situated individuals. That is, we consider scenarios where agents may have different outside options or simply not value the resource similarly. Analysis is difficult because the explanandum is now unclear. Just what are our intuitions about fairness once we move beyond the easy case? One popular thought is that fairness demands a 
division that corresponds to the so-called Nash bargaining solution (the division that maximizes the product of individual payoffs). We argue that there is a tentative case to be made for the Nash solution, gesturing to the discussion of bargaining in normative economics as well as experimental surveys eliciting the folk's intuitions about fairness. We then explore the evolution of bargaining behavior and find, somewhat surprisingly, that the Nash bargaining solution has substantial support. That is, evolution once again leads to divisions many recognize as fair.

\section{Fair bargains and the Nash solution}

Developed by John F. Nash in the 1950s, axiomatic bargaining theory addresses how agents should divide goods amongst themselves. In particular, the axiomatic approach stipulates what characteristics the outcome of bargaining should have. Before we discuss these features, a few definitions are in order. A bargaining problem consists of two entities: a feasible set and a disagreement point. Assuming a problem involving just two individuals, the feasible set refers to all utility pairs (outcomes) the agents could bring about. The disagreement point is an element of the feasible set and specifies what happens should agreement not be reached. Finally, a bargaining solution is a function which takes as input a bargaining problem and returns an element of the feasible set. The 'output' of a bargaining solution is taken to specify the agreement agents should make.

Theorists have studied the formal properties of several bargaining solutions. Famously, Nash introduced four axioms he thought any sensible bargaining solution should satisfy ${ }^{1}$. He then proved that one and only one solution satisfies all four axioms. This solution, which would later become known as the Nash bargaining solution, selects the division that secures the greatest product of individual utilities, sans the disagreement point. While the Nash solution is widely known, many competing solutions will be familiar to philosophers. David Gauthier defended a variant of the Kalai-Smorodinsky (KS) solution (Kalai \& Smorodinsky, 1975; Gauthier, 1986). This solution selects the utility pair which minimizes the maximum relative concession made by either bargainer. The egalitarian solution (equalize gain above the disagreement point) and the utilitarian solution (maximize the sum of payoffs) are also routinely discussed in bargaining theory (Thomson \& Lensberg, 2006).

Which of these solutions (if any) capture our intuitions about fairness in the bargaining problem? Unfortunately, the experimental literature on bargaining does not speak with one voice and tends to focus on behavior as opposed to the bargainers' fairness attitudes ${ }^{2}$. While some have suggested none of the standard solutions are apt, Binmore and co-authors (Binmore et al., 1993) found strong support for the Nash bargaining solution: experimental subjects gravitate away from the egalitarian and utilitarian solutions and move towards the Nash solution. Furthermore, by the end of Binmore's experiment subjects reported that agreements they made approximating the Nash solution struck them as 'fair.' Binmore's experiment is exceptional as it finds subjects tend to view agreements corresponding to the Nash solution as fair, giving us some reason to

\footnotetext{
${ }^{1}$ These are Pareto, Independence, Symmetry and Invariance.

${ }^{2}$ However, see (Schokkaert \& Overlaet, 1989) and (Yaari \& Bari-Hillel, 1984).
} 
favour the Nash solution. That said, while this experiment is very suggestive, the literature calls out for more empirical work documenting the folks' understanding of a fair bargain.

While experimental evidence is mixed, a tentative consensus exists in normative economics: the Nash solution is taken by many to be the fair and rational solution to the bargaining problem (Binmore, 2005; Moehler, 2010; Gauthier, 1993). For instance, after surveying various bargaining solutions in his book Equity, Peyton Young concludes that when it comes to fair bargains, the Nash solution is the 'most satisfactory' solution on offer (Young, 1995). Even David Gauthier, who famously attacked the Nash bargaining solution, eventually came to endorse it (Gauthier, 1993). The intuitions of the experts appear to coalesce around the Nash solution.

Assuming the Nash solution does in fact strike many as intuitive, is a compelling evolutionary explanation available? We survey the literature on the evolution of bargaining before turning to our results.

\section{Prior work on the evolution of bargaining}

Skyrms and Sugden independently consider the evolutionary dynamics of bargaining in the miniNash demand game where the position of the bargainers are symmetric (Sugden, 1986; Skyrms, 1996). Evolutionary forces push the population to the equal split under a variety of circumstances (see Alexander, 2007 for an overview). While illuminating, most evolutionary work on bargaining focuses on the mini-Nash demand game and thus assumes a strong kind of equality holds between bargainers (see O'Connor and Bruner, 2015 for an exception). While game-theoretical models always involve false assumptions, this idealization is particularly problematic because almost all sensible theories of fair division make the same recommendation when bargainers are symmetrically-situated. That is, the four solutions discussed earlier all recommend the equal split in the mini-Nash demand game. While evolution leads to the equal split, this could be because evolution tracks the Nash solution. If so, evolution would not result in the equal split in those games where the egalitarian and Nash solutions are distinct.

Little effort has been made to explore the evolution of behavior in more complicated bargaining scenarios where bargaining solutions do not coincide. Surprisingly, Skyrms discusses, albeit briefly, asymmetric bargaining games in the Postscript to the Evolution of the Social Contract. He considers two asymmetric bargaining problems and uses the replicator dynamic to determine the likelihood of various bargaining solutions. He finds in both games that the Nash solution is the modal outcome. Writing twenty years later, Peter Vanderschraaf correctly points out that while the Nash, KS and utilitarian divisions are mutually distinct in the games considered by Skyrms, the Nash and egalitarian bargaining solutions coincide (Vanderschraaf, 2018a,b). This motivates Vanderschraaf to analyze a handful of bargaining games where the egalitarian and Nash solution are distinct. Overall, Vanderschraaf's analysis indicates that the egalitarian solution may become 'prominent because these equilibria tend to be attracting points.' (2018, 175) 
Yet Vanderschraaf's results do not generalize beyond the select games he discusses. [Redactedfor-review] investigates a different (and much larger) class of bargaining games and found evolution favors the Nash bargaining solution (supporting Skyrms' original finding). Yet [Redacted's] analysis - while more systematic than past work on the topic - focuses exclusively on versions of the mixed-motive game known as hawk-dove. In what follows, we provide a more detailed and thorough analysis of the bargaining problem than has been hitherto offered. Looking at a variety of different games, we find that under certain conditions the Nash solution is likely to emerge, while under other conditions the KS solution is privileged. We contend the evolutionary significance of the Nash and KS solution hinges on a hitherto neglected distinction between comprehensive and non-comprehensive (Section 4) feasible bargaining sets.

Before turning to our evolutionary analysis, we briefly introduce, for illustrative purposes, a concrete bargaining problem. The game we introduce comes from Richard Braithwaite's inaugural lecture as Chair of Moral Philosophy at Cambridge and involves two musicians, Matthew and Luke (fittingly, we refer to this as the Matthew-Luke game). Matthew and Luke live in the same building. Both tenants desire to practice their instruments but due to the paperthin wall separating them, both prefer to play while the other is silent. This situation can be represented as a two-by-two game (Table 1a). Yet the strategic form representation is misleading as it suggests there are only two sensible solutions: Luke plays while Matthew listens, or vice versa. In fact, there are a continuum of pareto efficient conventions they could agree to. For instance, they could commit to the following plan of action: Matthew plays Monday, Tuesday, Wednesday, Luke plays Thursday, Friday and Saturday, and on Sunday they alternate play each week. Likewise, Matthew could play weekdays and Luke on the weekend. Following Braithwaite, we can represent these possibilities as the convex hull of the two-by-two game (Figure 1). This figure represents all conventions that Matthew and Luke can bring about (that is, all possible mixtures of the four base outcomes from Table 1a). The point in Figure 1 labelled $A$ corresponds to the scenario where Matthew takes the weekdays and Luke plays only on the weekend. If we follow Braithwaite and assume at the disagreement point both play their instruments, we can easily identify our four bargaining solutions. All solutions are efficient, but which (if any) do we expect Matthew and Luke to agree to?

Vanderschraaf analyses this game and, as mentioned, finds that under an adaptive dynamic the egalitarian solution is likely. Yet, as Vanderschraaf himself notes, this is merely suggestive. One bargaining problem is not enough to establish the evolutionary significance of the egalitarian solution. In what follows, we examine five large classes of bargaining scenarios for a total of 5,000 distinct bargaining problems. We find that while some specific strategic scenarios do favour the egalitarian solution, in most circumstances an adaptive dynamic pushes the population toward the Nash bargaining solution, or, in certain cases, the KS solution. 


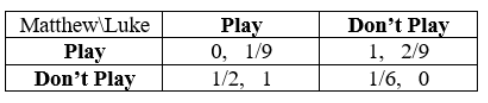

(a)

\begin{tabular}{|c|c|c|}
\hline & Defect & Cooperate \\
\hline Defect & 2,2 & 4,1 \\
\hline Cooperate & 1,4 & 3,3 \\
\hline
\end{tabular}

(c)

\begin{tabular}{|c|c|c|}
\hline & Play & Don't Play \\
\hline Play & 1,1 & 4,3 \\
\hline Don't Play & 3,4 & 2,2 \\
\hline
\end{tabular}

(e)

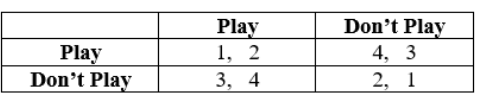

(b)

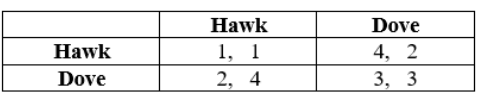

(d)

\begin{tabular}{|c|c|c|}
\hline & Play & Don't Play \\
\hline Play & 2,2 & 4,3 \\
\hline Don't Play & 3,4 & $1, \quad 1$ \\
\hline
\end{tabular}

(f)

Table 1: Cardinal Matthew-Luke game (a), and five ordinal two-player games: the ordinal Matthew-Luke game (b), the prisoner's dilemma (c), hawk-dove (d) and two versions of the battle of the sexes (e-f). We refer to these five ordinal games as OML, PD, HD, BoS-1 and BoS2 , respectively.

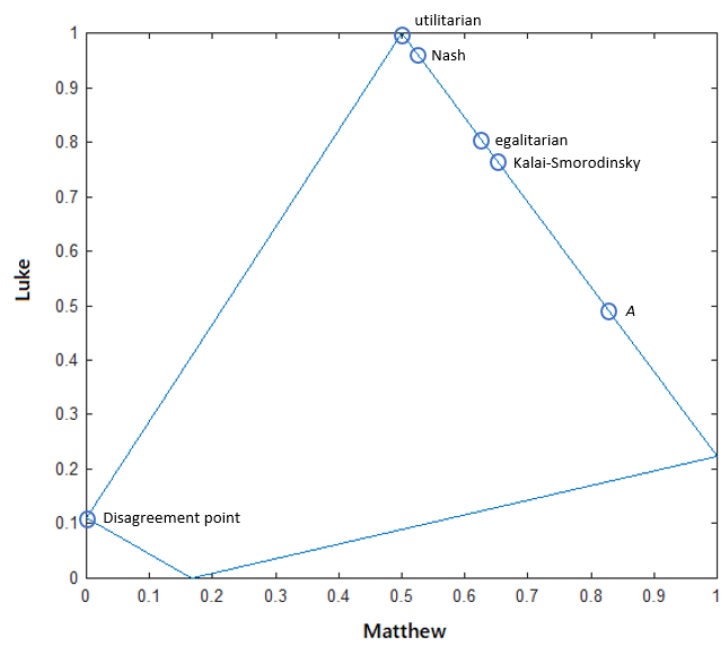

Figure 1: Bargaining problem based on the Matthew-Luke game.

\section{Prisoners' dilemma, hawk-dove and the battle of the sexes}

The Matthew-Luke game is an asymmetric battle of the sexes. In the one-shot version of the game, both want to coordinate on a pure Nash equilibrium, but there is disagreement as to which equilibrium is best, and neither fares well in the case of miscoordination. This strategic scenario is represented in ordinal payoffs in Table $1 \mathrm{~b}$, and we refer to this as the Ordinal Matthew-Luke game (OML). Braithwaite's original game can be viewed as an instantiation (or 'cardinalization') of the OML game, and it is possible to generate other instantiations of OML. We outline a simple method for cardinalizing familiar ordinal two-person games. This 
cardinalization method allows us to then explore the evolution of behavior in thousands of distinct games.

Cardinalization proceeds as follows. We construct a cardinal utility function for row player by making four draws from the uniform distribution spanning the unit interval ${ }^{3}$. We then assign these numbers to outcomes to match the ordinal ranking (row player's most-preferred outcome is matched with the highest of the four draws, etc.). We construct column player's cardinal utility function similarly. Once both individuals have cardinal utility functions, we can identify divisions corresponding to the various bargaining solutions. Furthermore, this cardinalization method allows us to construct hundreds of distinct versions of the OML game. As we will see, while these cardinal games are all instances of the same ordinal two-person game, the modal evolutionary outcome is not always the same. In some cardinalizations the Nash bargaining solution is privileged but, as Vanderschraaf observed, in other cardinalizations the egalitarian solution is likely to emerge. To determine the overall evolutionary significance of our four bargaining solutions, we look at 1,000 instances of the OML game. We also examine other ordinal games such as the prisoner's dilemma (PD), hawk-dove (HD), and two other versions of the battle of the sexes $(\mathrm{BoS})$ (see Table 1$)$.

One final qualification before our simulation results. In bargaining theory, it is common to assume that the feasible set is comprehensive. A set is comprehensive if for any utility pair $u=$ $\left(u_{1}, u_{2}\right)$, if $u$ is in the feasible set, then $\hat{u}=\left(u_{3}, u_{4}\right)$ is also in the feasible set so long as $u_{1} \geq u_{3}$ and $u_{2} \geq u_{4}$. That is, if $u$ is in the feasible set, then the feasible set also includes all utility pairs 'below and to the left' of $u$. Importantly, the feasible set must often be extended to ensure comprehensiveness. Comprehensiveness captures the fact that a bargainer has the right to destroy the good she secures in the course of bargaining. Since it is sensible to think individuals have the option of 'burning' utility, comprehensiveness is often assumed in the literature, and we begin our evolutionary analysis by considering bargaining problems with an (extended) comprehensive feasible set before then turning to problems that are not comprehensive. As mentioned, we find that comprehensiveness affects the evolutionary analysis, tilting the scales in favour of the Nash solution.

\section{Simulation set-up}

We now discuss our evolutionary simulations. For each distinct bargaining problem, we study the evolution of behavior with use of the replicator dynamic, a popular model of biological and cultural evolution ${ }^{4}$. We assume a "claim precision" of 1/100, meaning there are a total of 101 evenly spaced strategies (demands) spanning the unit interval ${ }^{5}$. If the demands made by the two agents are compatible (joint-claims are contained in the feasible set), both agents receive their

\footnotetext{
${ }^{3}$ We recover qualitatively similar evolutionary results for a beta $(\operatorname{Beta}(3,3))$ distribution.

${ }^{4}$ In particular we utilize the so-called two-population replicator dynamic. Using the game from Figure 1 as an example, members from one population play as "Matthew" and interact with members of the opposing population, who always play as "Luke".

${ }^{5}$ Vanderschraaf has a similar model but uses the learning dynamic fictitious play.
} 
claims; if not, the disagreement point obtains. We assume the disagreement point corresponds to the scenario where both agents are 'greedy' and play Hawk in the HD, Defect in the PD, or pursue their most preferred cooperative convention in the BoS.

For each of our five ordinal games (Table 1) we consider 1,000 distinct cardinalizations. For each cardinal bargaining problem, we run a total of 100 computer simulations and record the final state of the population for each simulation run. This allows us to determine - for each distinct bargaining problem - the size of the basin of attraction of a particular solution (that is, the proportion of simulations that result in a division that coincides with that solution). Since evolution does not always produce divisions that perfectly match our four bargaining solutions, we say a simulation run 'counts' as the Nash solution (for instance) if the end state of the simulation is within a certain distance, $r$, of the division recommended by the Nash solution ${ }^{6}$.

For a detailed picture of the evolution of bargaining behavior, we draw on three different measures and introduce them with reference to the Nash solution. First, we track the average size of the basin of attraction of the Nash solution. Second, we calculate the average distance (in Euclidean space) between the Nash solution and the endpoint of the evolutionary process. This gives us a sense of whether bargaining behavior is in the vicinity of the Nash solution. Finally, we record the number of bargaining games for which the basin of attraction for the Nash solution is greater than $50 \%$. This last measure provides us with a sense of how often the Nash solution is something like a global attractor.

\section{Comprehensive bargaining problems}

All five ordinal games yield similar qualitative results: the Nash solution is often the modal outcome and secures the largest average basin of attraction of our four bargaining solutions (Table 2). With respect to the average size of the basin of attraction, the Nash solution is followed by the utilitarian solution, the KS solution and, finally, the egalitarian solution. Furthermore, for the majority of distinct bargaining problems $(56.3 \%)$ it is the case that the size of the basin of attraction of the Nash solution exceeds 50\%. This is to say: evolved behavior is very likely to coincide with the Nash solution in many bargaining scenarios. Finally, even when the population does not end up at the Nash solution, bargaining behavior is often in the vicinity of the Nash solution: the average distance between evolved bargaining behavior and the Nash solution was 0.043. Compared to the utilitarian (0.097) and egalitarian solution (0.186), evolution has a strong tendency to produce divisions in the neighbourhood of the Nash solution.

Note that the utilitarian solution appears to do somewhat well, securing a large basin of attraction in many distinct bargaining problems $(47.2 \%)$. This is due in large part to the fact that in most bargaining problems (58.6\%) the Nash and utilitarian solutions coincide. In those cases where the Nash and utilitarian solutions are distinct, there is a tendency for evolution to push the population toward the Nash solution. Consider, for instance, Figure 2, which displays the relative size of the basin of attraction for a particular cardinalization of the PD. Note that in this

${ }^{6}$ This technique was also employed by [Redacted-for-review]. We consider $r=0.02$. 
bargaining problem our four solutions are mutually distinct and the distribution of bargaining outcomes of the evolutionary process is centered around the Nash bargaining solution. This is by no means atypical: in bargaining scenarios where the various solutions diverge, simulation results tend to cluster around the division corresponding to the Nash solution. However, as we see in the next section, this is not always the case. If the feasible set is not comprehensive, then the distribution of evolutionary outcomes is often anchored around the KS solution.

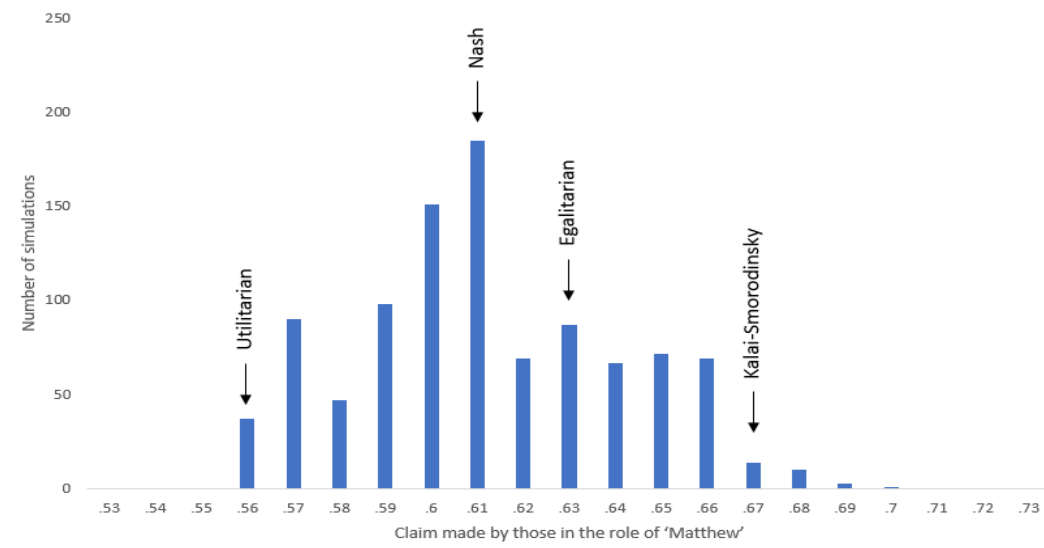

Figure 2: Distribution of bargaining outcomes of the evolutionary process for a comprehensive bargaining problem.

\begin{tabular}{|l|l|r|r|r|r|r|r|}
\hline \multicolumn{2}{|c|}{} & $\boldsymbol{P D}$ & $\boldsymbol{H D}$ & $\boldsymbol{B o S}-1$ & BoS-2 & OML & Average \\
\hline \multirow{3}{*}{ Nash } & Basin & 0.615 & 0.4537 & 0.6396 & 0.7286 & 0.6218 & 0.61174 \\
\cline { 2 - 8 } & Distance & 0.0374 & 0.051 & 0.052 & 0.0267 & 0.0486 & 0.04314 \\
\cline { 2 - 8 } & Global & 0.621 & 0.312 & 0.638 & 0.70 & 0.544 & 0.563 \\
\hline \multirow{3}{*}{ KS } & Basin & 0.4251 & 0.2716 & 0.4081 & 0.4053 & 0.4194 & 0.3859 \\
\cline { 2 - 8 } & Distance & 0.0586 & 0.08 & 0.0741 & 0.0542 & 0.0675 & 0.06688 \\
\cline { 2 - 8 } & Global & 0.37 & 0.157 & 0.308 & 0.328 & 0.335 & 0.299 \\
\hline \multirow{3}{*}{ Egalitatarian } & Basin & 0.3459 & 0.2882 & 0.4906 & 0.6615 & 0.5359 & 0.46442 \\
\cline { 2 - 8 } & Distance & 0.1172 & 0.1484 & 0.092 & 0.0443 & 0.0845 & 0.09728 \\
\cline { 2 - 8 } & Global & 0.362 & 0.297 & 0.53 & 0.662 & 0.511 & 0.472 \\
\hline & Basin & 0.2409 & 0.1012 & 0.0817 & 0.0883 & 0.1028 & 0.12298 \\
\cline { 2 - 8 } & Distance & 0.1184 & 0.1826 & 0.2129 & 0.1917 & 0.2256 & 0.18624 \\
\cline { 2 - 8 } & Global & 0.218 & 0.039 & 0.043 & 0.065 & 0.092 & 0.091 \\
\hline
\end{tabular}

Table 2: Results of simulations. For each ordinal game (column) and each of our four bargaining solutions (row), we determine average size of the basin of attraction (Basin), average distance from solution to endpoint of the simulation (Distance) and proportion of cardinal games where the basin of attraction for the bargaining solution was greater than $50 \%$ (Global).

\section{Non-comprehensive bargaining problems}

Recall that a bargaining problem is comprehensive if, for any utility pair in the feasible set, all utility pairs 'below and to the left' of this focal utility pair are also members of the feasible set. Intuitively, this means that individuals are free to dispose of utility as they see fit. While we have suggested it is reasonable to extend the feasible set to ensure a comprehensive bargaining problem, not all theorists assume comprehensiveness. Vanderschraaf, in his dynamic analysis of bargaining, only examines non-comprehensive bargaining problems. What effect does this have 
on the evolution of behavior? In this section we conduct an evolutionary analysis like the one provided above with the crucial caveat that we do not extend the feasible set to ensure comprehensiveness.

We see that this alteration heavily tilts the scales in favour the KS solution. As Table 3 indicates, KS now secures the largest average basin of attraction of all four solutions. Even when behavior does not coincide with the KS solution, evolution tends to select divisions in the neighbourhood of the KS solution. Furthermore, in many bargaining problems the size of the basin of attraction of the KS solution is well above $50 \%$, meaning the KS solution is extraordinarily likely in many strategic circumstances. To better visualize this, Figure 4 displays the distribution of evolutionary outcomes for a cardinalization of the OML game displayed in Figure 3. Note that the distribution is centered around the KS solution. Although Figure 4 pertains to just one bargaining problem, the distribution is nonetheless representative of a larger trend.

Why does evolution lead to the KS solution when the bargaining problem is no longer comprehensive? Consider a bargaining problem based on the Matthew-Luke game (Figure 3). Note that the Nash solution is at the edge of the pareto frontier ${ }^{7}$. This is no coincidence: the Nash solution is often near the edge of the pareto frontier for bargaining problems based on the HD or BoS. This fact has a surprising consequence. If Matthew aims to bring about the Nash solution but overshoots (he demands an amount greater than what is consistent with Nash), then the joint-claim will lie outside of the feasible set. Yet what if Matthew undershoots the Nash solution? Since the bargaining problem is not comprehensive, demanding an amount less than what is consistent with the Nash solution can also result in a joint-claim outside the feasible set. Thus, aiming for the Nash solution is especially risky, and, as a result, bargaining behavior will not settle on the Nash solution. Figure 4 illustrates this point, focusing once again on the bargaining problem from Figure 3. The distribution of bargaining outcomes of the evolutionary process for the extended comprehensive game is centered at the Nash solution. If undershooting the Nash is not penalized, evolution tends to produce divisions consistent with the Nash solution. The center of the distribution shifts to the KS solution if we do not extend the feasible set to ensure comprehensiveness. Since the Nash solution carries risk, evolution pushes the population to the 'safe' KS solution in the middle of the pareto frontier.

\section{Discussion}

We found that an adaptive dynamic often - though not always - results in the Nash solution. As suggested in Section 2, there is some evidence to support the claim that the Nash solution captures our fairness intuitions. If true, we have established that evolution tends to bring about fair bargains.

Yet whether the modal outcome corresponds to the Nash or KS solution hinges on the question of comprehensiveness. If individuals are allowed to burn utility then the Nash solution is favoured. Our findings highlight the importance of this often-overlooked distinction in

\footnotetext{
${ }^{7}$ The frontier is the set of pareto efficient bargains.
} 
bargaining theory, and hopefully future work will be mindful of the effect comprehensiveness has on evolutionary accounts of behavior.

While our analysis is more systematic than prior work, there still is much to do. First, it is unclear whether results on bargaining hold with the addition of a third or fourth bargainer. There is some reason to think the evolutionary prospects of the KS solution may be negatively affected, as the KS division is not always pareto efficient when more than two bargainers are involved. Second, we explored five different classes of ordinal two-person games, but an even more systematic investigation is within reach. Bruns (2015) provides a topology of two-person games and, by our count, this taxonomy indicates there are an additional 23 ordinal games of interest. While many avenues have yet to be explored, we have made modest progress toward a richer evolutionary account of our sense of fairness.

\begin{tabular}{|l|l|r|r|r|r|r|r|}
\hline \multicolumn{2}{|c|}{} & $\boldsymbol{P D}$ & $\boldsymbol{H D}$ & $\boldsymbol{B O S}-1$ & $\boldsymbol{B o S}-2$ & $\boldsymbol{O M L}$ & Average \\
\hline \multirow{3}{*}{ Nash } & Basin & 0.6497 & 0.3327 & 0.3338 & 0.2228 & 0.2982 & 0.36744 \\
\cline { 2 - 8 } & Distance & 0.0736 & 0.0685 & 0.0577 & 0.1011 & 0.0629 & 0.07276 \\
\cline { 2 - 8 } & Global & 0.649 & 0.221 & 0.312 & 0.155 & 0.218 & 0.311 \\
\hline \multirow{3}{*}{ KS } & Basin & 0.4251 & 0.3983 & 0.5846 & 0.5394 & 0.5884 & 0.50716 \\
\cline { 2 - 8 } & Distance & 0.0974 & 0.053 & 0.0365 & 0.0605 & 0.0348 & 0.05644 \\
\cline { 2 - 8 } & Global & 0.338 & 0.21 & 0.629 & 0.501 & 0.587 & 0.453 \\
\hline \multirow{3}{*}{ Egalititarian } & Basin & 0.4088 & 0.1676 & 0.1541 & 0.1571 & 0.1834 & 0.2142 \\
\cline { 2 - 8 } & Distance & 0.1565 & 0.1714 & 0.1112 & 0.1342 & 0.1067 & 0.136 \\
\cline { 2 - 8 } & Global & 0.45 & 0.148 & 0.152 & 0.109 & 0.135 & 0.1988 \\
\hline & Basin & 0.27 & 0.1886 & 0.1457 & 0.0957 & 0.075 & 0.155 \\
\cline { 2 - 8 } & Distance & 0.1459 & 0.142 & 0.1518 & 0.2016 & 0.2258 & 0.17342 \\
\cline { 2 - 8 } & Global & 0.229 & 0.096 & 0.12 & 0.047 & 0.041 & 0.1066 \\
\hline
\end{tabular}

Table 3: Results of simulations for non-comprehensive problems.

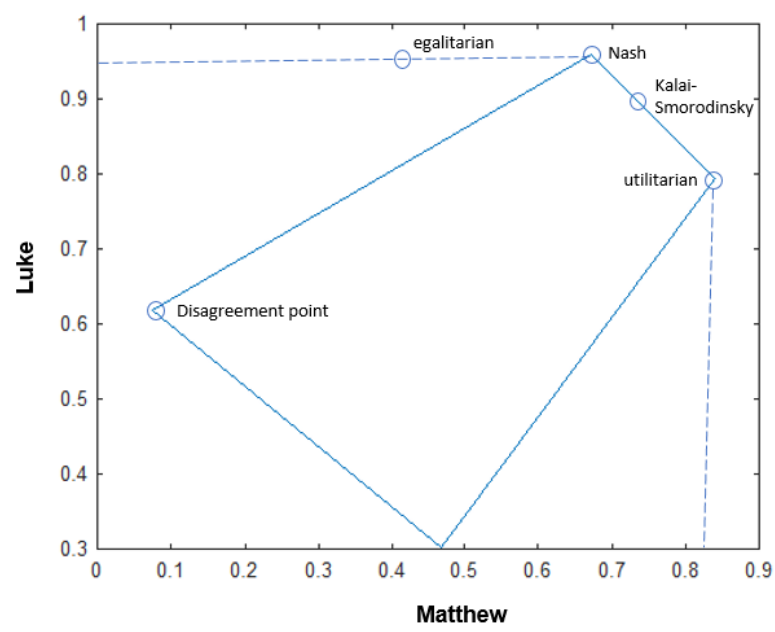

Figure 3: Bargaining problem based on the OML. Dotted lines indicate the extended comprehensive feasible set. 


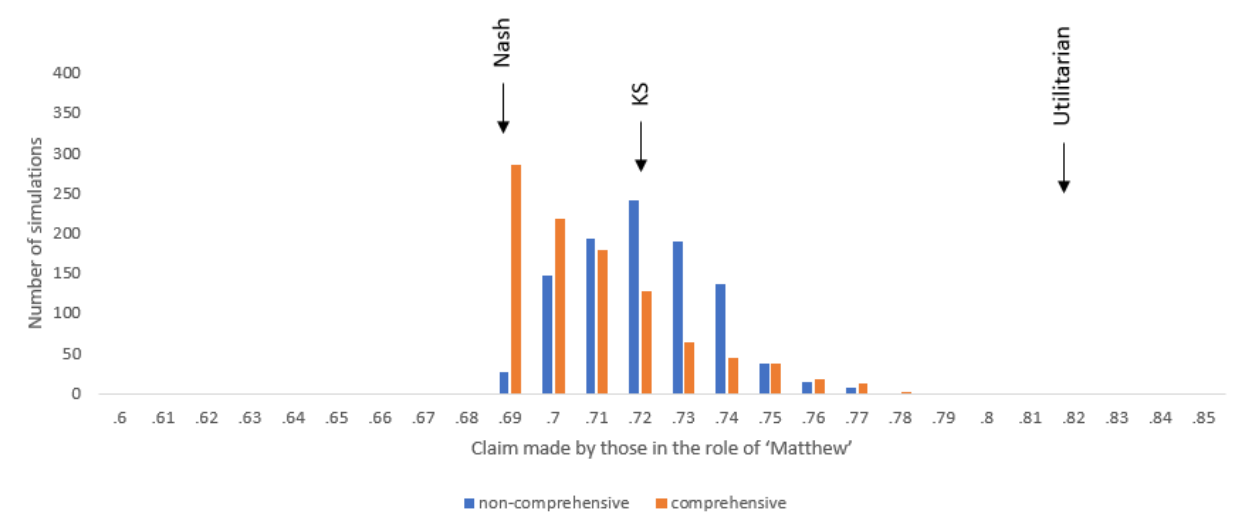

Figure 4: Distribution of bargaining outcomes of the evolutionary process for the bargaining problem displayed in Figure 3 (non-comprehensive in blue and comprehensive in orange).

\section{References}

Alexander, J. (2007). The Structural Evolution of Morality. Cambridge University Press.

Binmore, K. (2005). Natural Justice. Oxford University Press.

Binmore, K., Swierzbinski, J., Hsu, S., \& Proulx, C. (1993). Focal points and bargaining. International Journal of Game Theory, 381-409.

Braithwaite, R. (1955). Theory of Games as a Tool for the Moral Philosopher. Cambridge University Press.

Bruns, B. (2015). Names for Games. Games, 495-520.

Ernst, Z. (2005). A plea for asymmetric games. Journal of Philosophy, 109-125.

Gauthier, D. (1986). Morals by Agreement. Oxford University Press.

Gauthier, D. (1993). Uniting separate persons. Rationality, Justice and the Social Contract, pp. 176-192.

Kalai, E., \& Smorodinsky, M. (1975). Other solutions to Nash's bargaining problem. Econometrica, 513518.

Moehler, M. (2010). The (stabilized) Nash bargaining solution as a principle of distributive justice. Utilitas, 447-473.

Nydegger, R. V., \& Owen, G. (1974). Two-person bargaining: an experimental test of the Nash axioms. International Journal of Game Theory, 239-249.

O'Connor, C., \& Bruner, J. (2019). Dynamics and diversity in epistemic communities. Erkenntnis, 101119.

review, R. f. (n.d.).

Schokkaert, E., \& Overlaet, B. (1989). Moral intuitions and economic models of distributive justice. Social Choice and Welfare, 19-31. 
Skyrms, B. (1996). Evolution of the Social Contract. Cambridge University Press.

Sugden, R. (1986). The Economics of Rights, Cooperation and Welfare. Blackwell.

Thomson, W., \& Lensberg, T. (2006). Axiomatic Theory of Bargaining with a Variable Number of Agents. Cambridge University Press.

Vanderschraaf, P. (2018). Learning bargaining conventions. Social Philosophy and Policy, 237-263.

Vanderschraaf, P. (2018). Strategic Justice. Oxford.

Yaari, M., \& Bari-Hillel, M. (1984). On dividing justly. Social Choice and Welfare, 1-24.

Young, P. (1995). Equity. Princeton University Press. 